Hautarzt 2018 $69: 528-535$

https://doi.org/10.1007/s00105-018-4204-8

Online publiziert: 6. Juni 2018

(c) Der/die Autor(en) 2018

CrossMark

\section{H. Kittler}

Universitätsklinik für Dermatologie, Medizinische Universität Wien, Wien, Österreich

\section{Dermatoskopisch-pathologische Korrelation melanozytärer Hautläsionen}

Eine rezente Studie konnte zeigen, dass die dermatopathologische Untersuchung melanozytärer Läsionen fehlerbehaftet ist [6]. Wenn mehrere Untersucher dieselbe Läsion beurteilen, kann es zu unterschiedlichen Befunden kommen, die von sicher gutartig bis $\mathrm{zu}$ sicher bösartig reichen. Sowohl falsch positive als auch falsch negative Befunde sind ein großes reales Problem. Die Dermatopathologie hat zwar das letzte Wort, hat aber nicht immer Recht. Das ist gerade für den Kliniker schwer zu akzeptieren, da das Fehlen einer letztgültigen Diagnose, der man uneingeschränkt vertrauen kann, sowohl beim Arzt als auch beim Patienten zu Verunsicherung führt.

\section{Diagnostischer Pragmatismus}

Ein möglicher Ausweg aus diesem Dilemma ist eine Strategie, die man als diagnostischen Pragmatismus bezeichnen könnte. Damit ist gemeint, dass die Diagnose selbst gar nicht mehr in den Vor- dergrund gestellt wird, sondern die Konsequenzen. Der Befunder stellt keine Diagnose mehr, sondern gibt dem Kliniker Handlungsanweisungen, die in 5 Klassen unterteilt werden [7]. Zum Beispiel lautet die Anweisung in der gutartigsten Klasse (Klasse 1): Selbst wenn die Läsion unvollständig entfernt wurde, ist keine Nachexzision erforderlich. Bei Läsionen der Klasse 2 sollte die Läsion zumindest ganz entfernt werden, aber ohne Sicherheitsabstand, während bei Läsionen der Klassen 3, 4 und 5 immer größere Sicherheitsabstände und vermehrt auch Zusatzuntersuchungen, wie z. B. die Sentinellymphknotenbiopsie in der Klasse 5, empfohlen werden. Es überrascht nicht, dass diese Strategie ihren Ursprung in jenem Land hat, in dem auch der philosophische Pragmatismus seine Heimat hat [8]. Dieses Vorgehen mag zwar einige praktische Probleme lösen, ob es aus erkenntnistheoretischer Sicht sinnvoll ist, In-situ-Melanome und Spitz-Nävi in eine Kategorie zu werfen, sei dahingestellt.

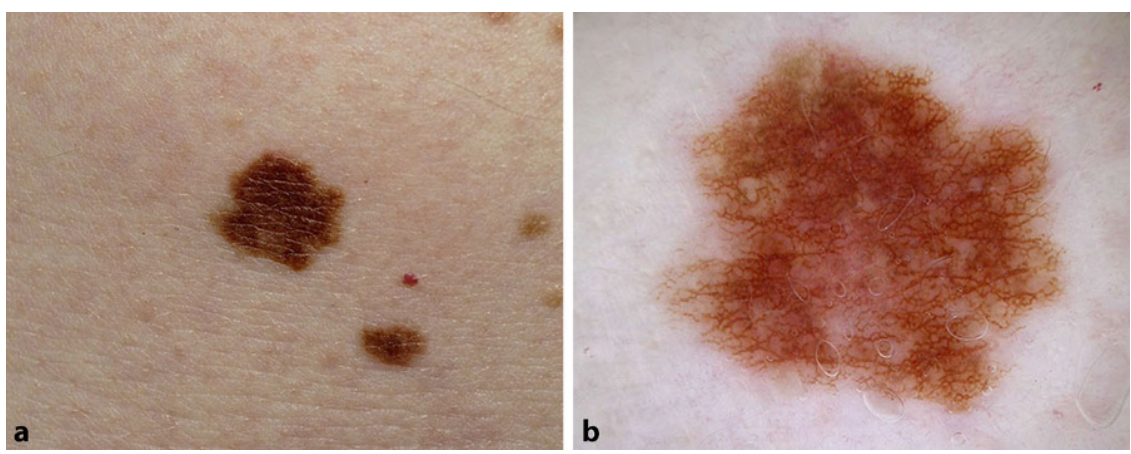

Abb. $1 \Delta$ Unbewaffnetes Auge vs. Dermatoskopie. a Ein Nävus, wie er mit dem freien Auge erscheint. In b ist derselbe Nävus dermatoskopisch dargestellt. Am rechten Bild kann man retikuläre Linien erkennen, die mit dem freien Auge unsichtbar sind. Die hypopigmentierten Zwischenräume korrelieren mit den dermalen Papillen, und die hyperpigmentierten Linien entsprechen den pigmentierten Reteleisten. (Aus [23], mit freundl. Genehmigung $\odot$ H. Kittler, alle Rechte vorbehalten. Dieser Inhalt ist nicht Teil der Open-Access-Lizenz.) 


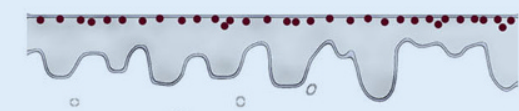

a

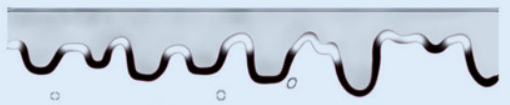

C

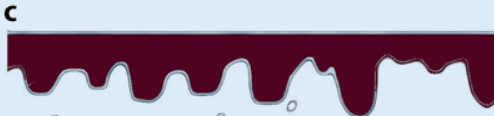

e

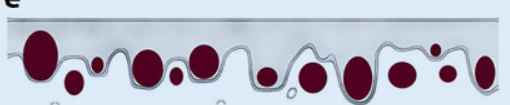

g

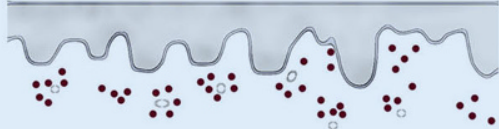

i

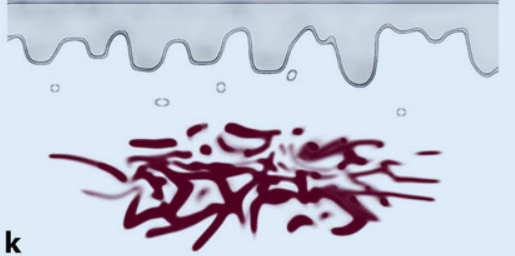

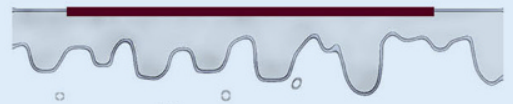

b

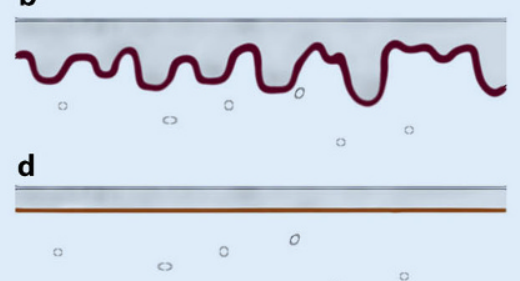

f

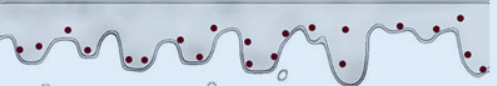

h

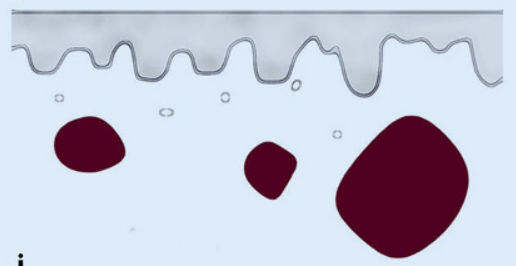

j

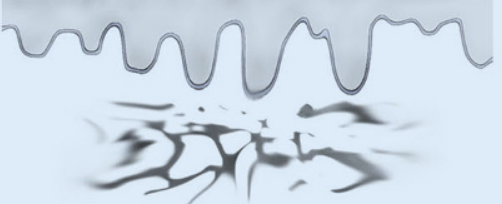

I

schen Untersuchungen eine theoretische Grenze. Selbst wenn wir jedes Basenpaar und jede Aminosäuresequenz eines Tumors kennen würden, könnten wir sein biologisches Verhalten nicht mit Sicherheit vorhersagen, wenn dieses Verhalten auch von der Tumorumgebung beeinflusst wird.

Im Gegensatz zu molekularbiologischen Untersuchungen werden klinische Befunde bei problematischen Läsionen oft ignoriert oder weniger ernst genommen. Zwar spielen klinische Angaben wie Lokalisation und Alter des Patienten in speziellen Fällen implizit eine gewichtige Rolle [11], doch werden diese Informationen selten explizit bei der Befundung berücksichtigt. Es gibt zwar einige Dermatopathologen, die das klinische oder dermatoskopische Bild in ihre Beurteilung einfließen lassen [12], aber im Allgemeinen überwiegt nach wie vor eine gewisse Skepsis, ob diese Integration im Alltag überhaupt sinnvoll ist. Diese Skepsis wird auch dadurch gefördert, dass nur wenige Dermatopathologen mit dem klinischen oder dermatoskopischen Erscheinungsbild etwas anfangen können. Eine Expertise in diesen Bereichen ist jedoch Voraussetzung für eine sinnvolle Integration dieser Information [11].

\section{Dermatoskopie pigmentierter Läsionen}

Die Dermatoskopie ist eine nichtinvasive Untersuchungstechnik, bei der störende Reflexions- und Streuungsphänomene an der Hautoberfläche vermindert werden. Im Gegensatz zur Betrachtung mit dem freien Auge kann man mithilfe der Dermatoskopie auch tiefere Strukturen darstellen. Mit tieferen Strukturen sind v. a. Veränderungen im Bereich der dermoepidermale Junktionszone gemeint [12, 13]. Während histopathologische Schnitte ihren farblichen Kontrast durch artifiziell zugefügte Farbstoffe (z. B. Hämatoxylin und Eosin) erhalten, nutzt man in der Dermatoskopie das natürlich vorhandene Melanin. Melanin in der Epidermis erscheint dermatoskopisch braun oder schwarz. Die in der Dermatoskopie sichtbaren braunen retikulären Linien (auch Pigmentnetz genannt) korrespondieren mit der Pigmentierung im Bereich der

feinerte molekulare Methoden beseitigt wann. Die derzeitige Datenlage nicht immer mit der Biologie und somit mit der Dignität der Läsion [10]. Diese Untersuchungen haben sicher ihren Stellenwert, werden sich aber sehr wahrscheinlich auch nur als Teile des Puzzles herausstellen und nicht als Puzzlevorlage. Es ist durchaus wahrscheinlich, dass sich derselbe Tumor in einer anderen Umgebung unterschiedlich verhält. Das wiederum setzt auch molekularbiologi- 
dermoepidermalen Junktionszone, wobei die hypopigmentierten Zwischenräume den dermalen Papillen und die hyperpigmentierten Linien den Reteleisten entsprechen (•Abb. 1). Da Melanin in der Dermis dermatoskopisch grau oder blau erscheint, kann man auch anhand des Farbtons die Tiefenausdehnung grob abschätzen [14]. Der blaue Farbton blauer Nävi oder invasiver Melanome ist auf pigmentbeladene Melanozyten in der retikulären Dermis zurückzuführen. Im Prinzip kann jede dermatoskopische Struktur einem histopathologischen Korrelat zugeordnet werden (• Abb. 2), und somit ist es möglich, aus dem dermatoskopischen Bild die Pathologie abzuleiten und umgekehrt. Das kann sowohl für den Kliniker als auch für den Pathologen von großem Nutzen sein.

Man muss jedoch zugeben, dass die Dermatoskopie in erster Linie für oberflächlich gelegene Läsion geeignet ist, während dermale Prozesse nur mit Einschränkungen beurteilt werden können.

\section{》) Im Prinzip kann jede dermatoskopische Struktur einem histopathologischen Korrelat zugeordnet werden}

Das Haupteinsatzgebiet der Dermatoskopie ist die Untersuchung von Pigmentläsionen, in erster Linie von melanozytären Läsionen. Dabei ist wie in der Dermatopathologie die Asymmetrie ein wichtiges Kriterium zur Unterscheidung zwischen Nävus und Melanom [15]. Dermatoskopische Asymmetrie ergibt sich in erster Linie aufgrund einer ungleichmäßigen Verteilung von Melanin. Im Gegensatz zur Dermatopathologie, bei der nur einige wenige, ultradünne, vertikale Schnittebenen zur Verfügung stehen, beurteilt man in der Dermatoskopie die Gesamtläsion in der Horizontalebene, wobei das eigentliche Erscheinungsbild einem durch Absorption, Reflexion und Streuung von Licht bedingten Summeneffekt in übereinander geschichteten Ebenen entspricht. So gesehen enthält die Dermatoskopie mehr Information als ein pathologischer Schnitt.

Hautarzt 2018 -69:528-535 https://doi.org/10.1007/s00105-018-4204-8

(c) Der/die Autor(en) 2018

\section{H. Kittler}

\section{Dermatoskopisch-pathologische Korrelation melanozytärer Hautläsionen}

\section{Zusammenfassung}

Ohne Zweifel stellt die Dermatopathologie nach wie vor die wichtigste Untersuchung zur Dignitätsabklärung melanozytärer Läsionen dar, doch ist die dermatopathologische Befundung wie alle morphologischen Untersuchungen subjektiv gefärbt. Wie eine rezente Studie zeigen konnte, gibt es eine große Variabilität bei der Befundung melanozytärer Läsionen, und falsch positive und falsch negative Befunde sind verhältnismäßig häufig. Unabhängig vom Befunder besteht auch eine Limitation der Untersuchungsmethode selbst, und man muss sich damit abfinden, dass nicht alle melanozytären Läsionen anhand einer feingeweblichen dermatopathologischen Untersuchung zweifelsfrei als gutartig oder bösartig klassifiziert werden können. In jenen Fällen, wo das nicht gelingt, kann die Einbeziehung des dermatoskopischen Befundes die Diagnosestellung erleichtern. Eine dermatoskopisch-pathologische Korrelation ist jedoch nur dann möglich, wenn ein dermatoskopisches Bild zur Verfügung steht und wenn der Pathologe mit der Interpretation dermatoskopischer Strukturen vertraut ist. Eine dermatoskopisch-pathologische Korrelation ist nicht bei allen unklaren Läsionen hilfreich, sondern bevorzugt bei flachen pigmentierten Läsionen. In diesen Fällen jedoch ist der Informationsgewinn durch die Dermatoskopie wahrscheinlich größer als durch zusätzliche molekulare Untersuchungen.

Schlüsselwörter

Dermatopathologie - Dermatoskopie . Melanom · Nävi · Dermatoonkologie

\section{Dermatoscopic-pathological correlation of melanocytic skin lesions}

\section{Abstract}

There is no doubt that dermatopathology is the most important method to decide if a melanocytic lesion is benign or malignant; however, like most morphologic examinations, dermatopathology is subjective. A recent study demonstrated that the pathologic diagnosis of melanocytic skin lesions has a high variability. Reports with false-positive or false-negative diagnoses are relatively common. The pathologic examination of melanocytic lesions also has observerindependent limitations and one has to accept that some melanocytic lesions cannot be classified as benign or malignant with confidence by dermatopathology alone. If a confident diagnosis is not possible a dermatoscopic-pathologic correlation may be helpful. This, however, is only possible if dermatoscopic images are available and if the dermatopathologist knows how to interpret dermatoscopic structures. A dermatoscopic-pathologic correlation is not useful in all difficult melanocytic lesions but it should be considered in difficult flat pigmented lesions. In these cases dermatoscopy may provide even more important additional information than molecular findings.

\section{Keywords}

Dermatopathology - Dermoscopy/

Dermatoscopy · Melanoma · Nevi . Dermatooncology

\section{Vorteile der Integration dermatoskopischer Befunde}

Kann man anhand der histopathologischen Kriterien keine sichere Diagnose stellen oder steht die histopathologische Diagnose im Widerspruch mit der klinischen oder dermatoskopischen Diagnose, so kann es hilfreich sein, beide Untersuchungen zu integrieren. In einer Studie hat sich gezeigt, dass in manchen Fällen zusätzliche klinische Informationen oder das dermatoskopische Bild Dermatopathologen dazu veranlasst, die ursprünglich gestellte histopathologische Diagnose zu überdenken und zu revidieren [11]. Dabei ist bemerkenswert, dass Dermatopathologen, die auch als Dermatologen tätig sind, eher bereit sind, das dermatoskopische Bild in den Befund zu integrieren, als Pathologen, die keine klinische Erfahrung haben. Ausschlaggebend da- 

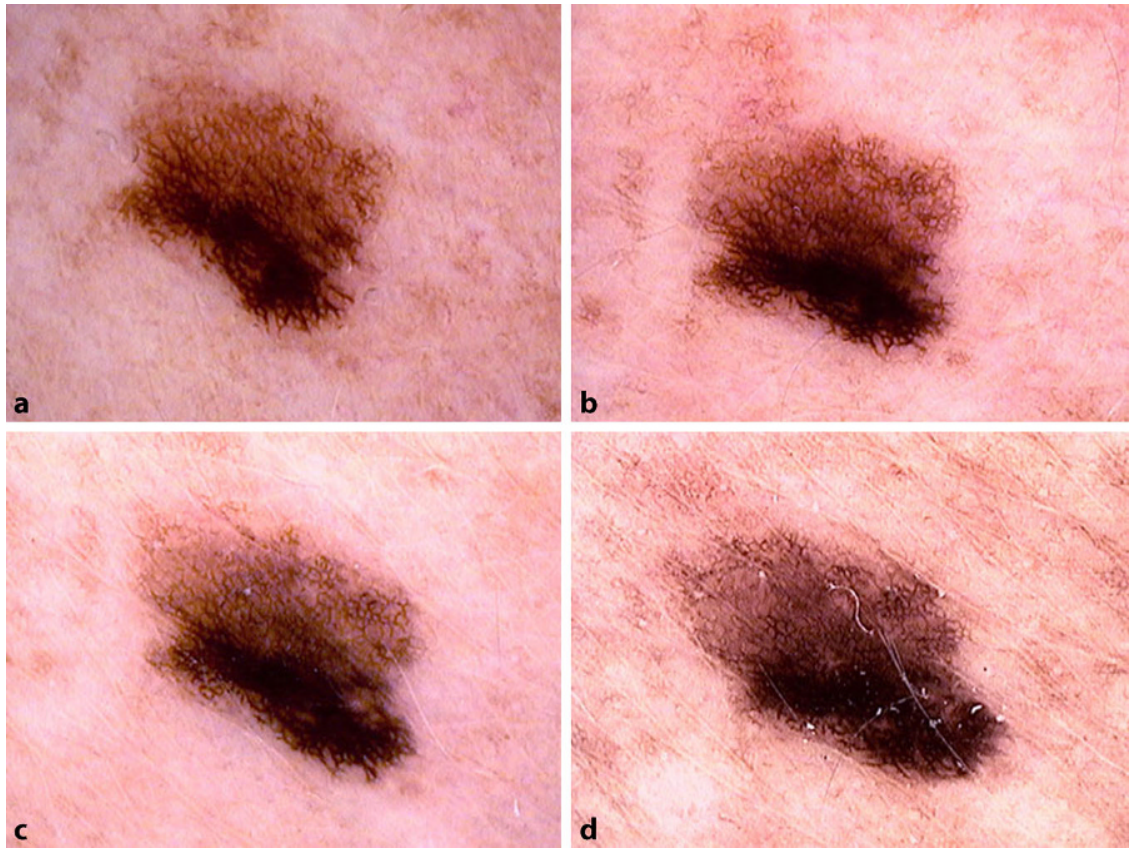

Abb. 3 ॥ Dermatoskopische Verlaufskontrolle. Melanozytäre Läsion bei einem über 60-jährigen Patienten mit langsamer exzentrischer Größenzunahme. Es handelt sich um digitale dermatoskopische Aufnahmen, die in 6-monatigen Abständen durchgeführt werden. Die Biologie dieser Läsion ist expansiv und spricht für ein Melanom, obwohl die Morphologie nicht so eindeutig erscheint. (Mit freundl. Genehmigung $\odot$ H. Kittler, alle Rechte vorbehalten. Dieser Inhalt ist nicht Teil der Open-Access-Lizenz.)

für ist wohl, dass die eine Gruppe Erfahrung in der Interpretation dermatoskopischer Bilder hat, die andere aber nicht und daher die Information, mit der sie nichts anfangen kann, ignoriert.

\section{Der einzige diagnostische Standard?}

Die Dermatopathologie untersucht ein Stück totes Gewebe. Ist eine melanozytäre Läsion einmal exzidiert, bleibt ihr biologisches Schicksal unklar. Die Dermatopathologie versucht anhand der Morphologie die Biologie vorherzusagen, was nicht immer zweifelsfrei gelingt. Es gibt jedoch Situationen, in denen man die Biologie direkt beobachten kann. Metastasierung beweist biologische Bösartigkeit. Wurde eine melanozytäre Läsion, die später metastasierte, ursprünglich als Nävus befundet, wird allgemein akzeptiert, dass die Biologie den dermatopathologischen Befund widerlegt und somit den eigentlichen diagnostischen Standard darstellt. Für den umgekehrten Fall gilt das natürlich nicht, da eine ausbleibende Metastasierung eine vorhandene, aber nicht realisierte Bösartig- keit einer vollständig entfernten Läsion nicht ausschließt. Metastasierung ist zum Glück nicht die einzige Beobachtung, die Rückschlüsse auf die Biologie der Läsion zulässt. Veränderung und Wachstum der Läsion selbst können mittels digitaler Dermatoskopie beobachtet werden. Wenn diese Veränderungen gewisse Charakteristika, wie z. B. asymmetrische Größenzunahme, aufweisen und wenn das Alter des Patienten einen wachsenden Nävus sehr unwahrscheinlich macht, dann kann auch die digitale Dermatoskopie als Indikator für die Biologie der Läsion herangezogen werden [16-18]. In - Abb. 3 ist eine melanozytäre Läsion bei einem über 60-jährigen Patienten dargestellt, die langsam an Größe zunimmt und dabei exzentrisch wächst. Es handelt sich um digitale dermatoskopische Aufnahmen, die standardmäßig bei der Verlaufskontrolle von Patienten mit multiplen Nävi durchgeführt werden. Eine benigne Diagnose, also ein Nävus, wäre biologisch und dermatoskopisch sehr unwahrscheinlich. In • Abb. 4 ist die Dermatopathologie dieser Läsion dargestellt. In der Übersicht sieht man zwar einige regelmäßige Nester im Bereich der der- moepidermalen Junktionszone, bei genauer Betrachtung jedoch zeigen sich in der Peripherie v. a. Einzelzellen, und in Zusammenschau mit dem dermatoskopischen Bild und der Biologie kann man die Läsion sicher als In-situ-Melanom diagnostizieren. Hätte man das dermatoskopische Bild bzw. die Verlaufsbilder nicht zur Verfügung, wäre die Diagnose nicht so klar. In diesem Fall erhöht also die Integration der Dermatoskopie die diagnostische Sicherheit.

\section{Praktische Integration: Wie und wann?}

Es soll nicht der Eindruck vermittelt werden, dass die Dermatopathologie melanozytärer Läsionen ohne Integration dermatoskopischer Befunde mangelhaft ist. Das ist im Allgemeinen nicht der Fall, denn die pathologischen Kriterien, die zur Diagnose herangezogen werden, haben sich über viele Jahre bewährt [19]. In der täglichen Praxis können die allermeisten melanozytären Läsionen auch ohne dermatoskopisch-pathologische Integration einwandfrei als Nävus oder als Melanom klassifiziert werden. Sinnvoll ist eine Integration des dermatoskopischen Befundes nur dann, wenn dermatopathologisch Zweifel bestehen. Wie oft Zweifel bestehen und wie groß die Zweifel sind, hängt natürlich vom Befunder ab. Zweifel wird oft als Unsicherheit fehlgedeutet. Der erfahrene Dermatopathologe kennt die Grenzen der Untersuchung und wird daher nicht der Illusion verfallen, dass seine eigenen Befunde über jeden Zweifel erhaben sind. Wenn Zweifel bestehen, sollten alle zusätzlichen Informationen ausgeschöpft werden. Man darf jedoch nicht übersehen, dass der dermatoskopische Befund nur in manchen Fällen hilfreich ist. Bei knotigen oder unpigmentierten Läsionen hilft die Dermatoskopie in der Regel nicht weiter. Die Dermatoskopie hat ihre Stärken in der Beurteilung flacher pigmentierter Läsionen der dermoepidermalen Junktionszone, tiefe dermale Prozesse hingegen sind mit dem Dermatoskop nur eingeschränkt beurteilbar. Ein gar nicht so seltenes, praktisch relevantes Beispiel wäre die Unterscheidung zwischen einem klassi- 


\section{Leitthema}
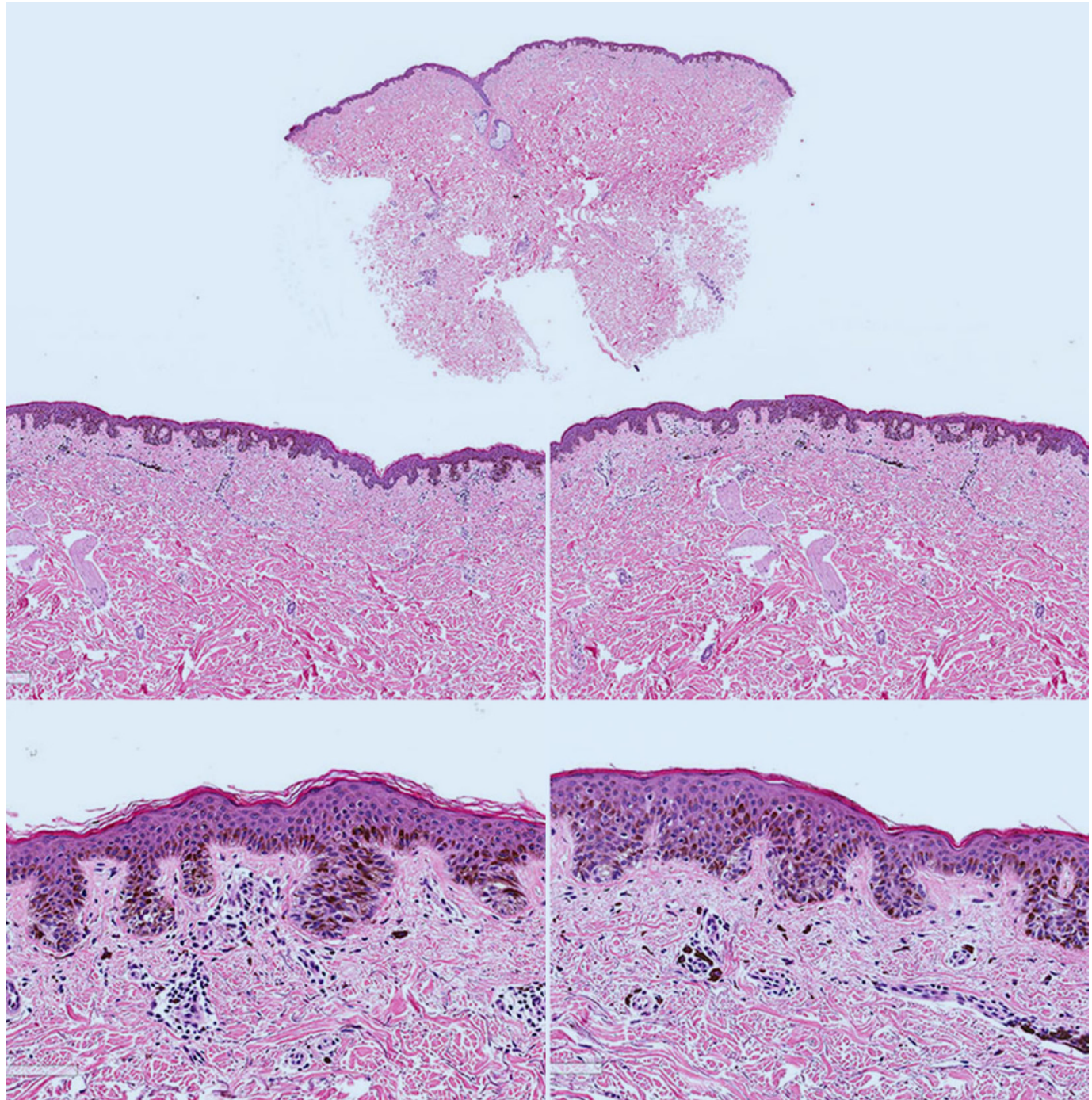

Abb. $4<$ Dermatoskopisch-pathologische Korrelation. Dermatopathologie der in $\bullet$ Abb. 3 dargestellten Läsion. In der Übersicht ist man geneigt, die Läsion als junktionalen Clark-Nävus („dysplastischer" Nävus) zu befunden, jedoch sieht man bei

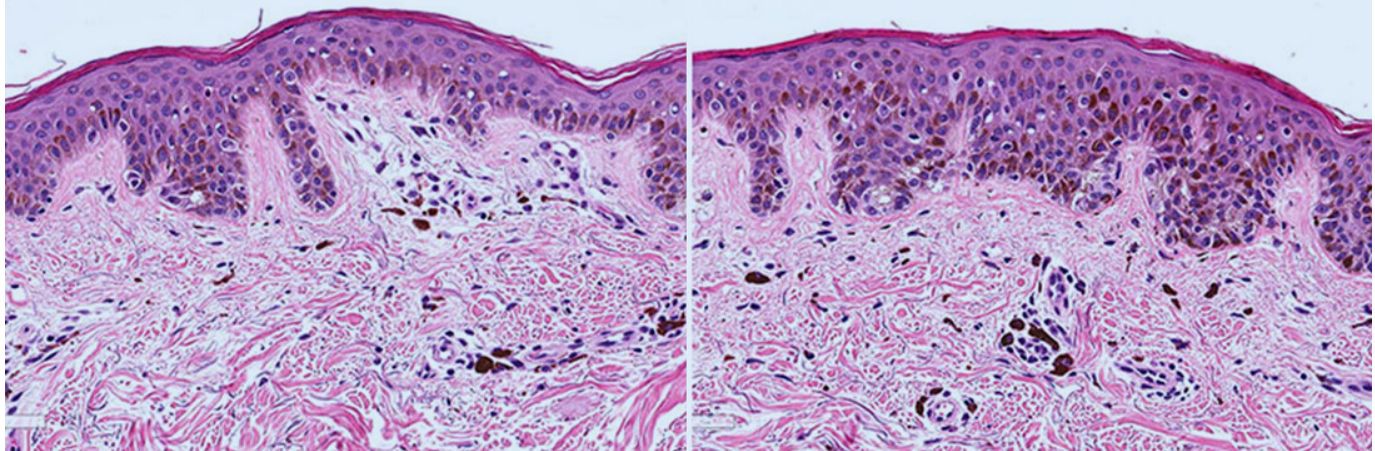
genauerer Betrachtung in der Peripherie überwiegend Einzelzellen, und in Zusammenschau mit den sequenziellen dermatoskopischen Bildern kann man die Läsion sicher als In-situMelanom diagnostizieren. (Mit freundl.Genehmigung (c) H. Kittler, alle Rechte vorbehalten. Dieser Inhalt ist nicht Teil der Open-AccessLizenz.)

schen unpigmentierten Spitz-Nävus und einem „spitzoiden“ Melanom [20]. In diesem Fall würde die Dermatoskopie nicht weiterhelfen, molekularbiologische Untersuchungen wie FISH oder CGH aber möglicherweise schon.

Auf der anderen Seite ist der Wert molekularbiologischer Untersuchungen bei In-situ-Melanomen äußerst fraglich. Bisher gibt es dazu auf jeden Fall keine Daten. Der dermatoskopische Befund jedoch kann bei In-situ-Melanomen, die ja in der Regel als flache unregelmäßig pigmentierte Läsionen in Erscheinung treten, die Sachlage klären. In • Abb. 5 ist ein In-situ-Melanom dargestellt, das derma- topathologisch leicht mit einem ClarkNävus (sog. „dysplastischer" Nävus) verwechselt werden kann. Die Integration des dermatoskopischen Bildes räumt jedoch alle Zweifel aus (• Abb. 6).

Somit beantwortet sich auch die Frage, ob die Integration des dermatoskopischen Bildes vor oder nach der Be- 

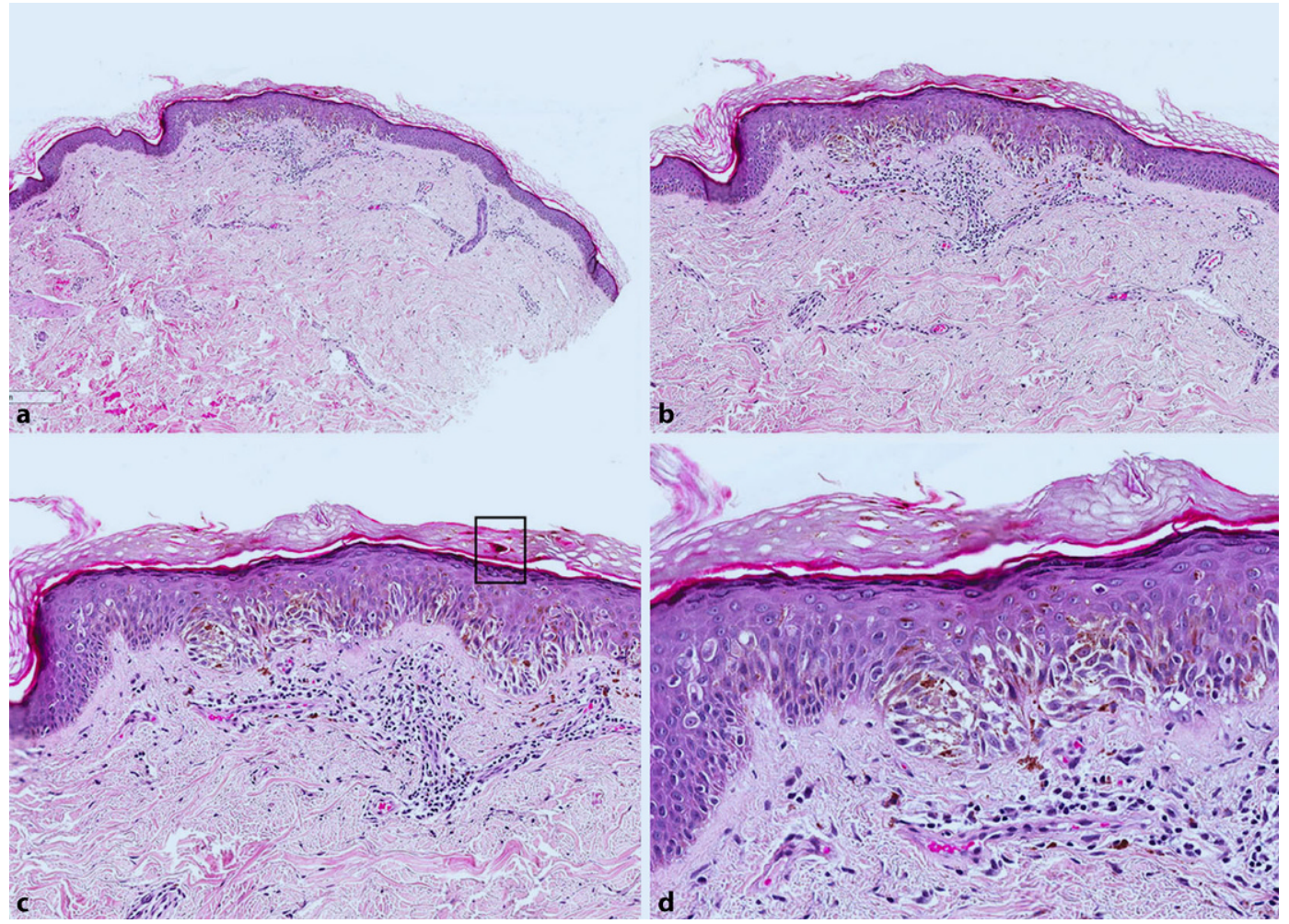

Abb. $5<$ In-situ-Melanom oder Nävus? Dermatopathologie einer junktionalen melanozytären Proliferation, die man aufgrund der Kleinheit der Läsion schwer einordnen kann. Man könnte die Läsion als junktionalen Clark-Nävus („dysplastischer" Nävus) befunden. Bei genauerer Betrachtung und v. a. bei Miteinbeziehung des dermatoskopischen Bildes (• Abb. 6) wird man sich aber doch für ein In-situ-Melanom entscheiden. Das schwarze Rechteck weist auf Melaninansammlungen im Stratum corneum hin (•Abb. 6). (Mit freundl. Genehmigung (c) H. Kittler, alle Rechte vorbehalten. Dieser Inhalt ist nicht Teil der Open-AccessLizenz.)
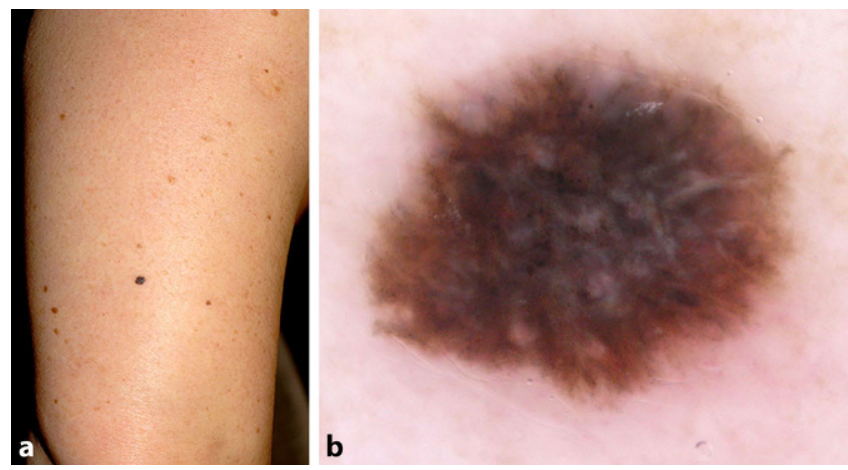

Abb. 6 A a, b Dermatoskopisch-pathologische Korrelation. a Klinisches Bild. b Dermatoskopisches Bild. Obwohl die Läsion sehr klein ist, ist sie dermatoskopisch unregelmäßig pigmentiert (",chaotisch“) und weist Melanomkriterien auf (schwarze Punkte in der Peripherie und graue Linien). Den schwarzen Punkten entsprechen die pigmentierten Melanozyten in höheren Epidermislagen und die Melaninansammlungen im Stratum corneum, die in • Abb. 5 zu sehen sind. (Mit freundl. Genehmigung () H. Kittler, alle Rechte vorbehalten. Dieser Inhalt ist nicht Teil der Open-Access-Lizenz.)

gutachtung des dermatopathologischen Schnittes erfolgen soll. Die Integration sollte immer nach der dermatopathologischen Begutachtung erfolgen und nur in jenen Fällen, in denen Zweifel bestehen und ein Informationsgewinn durch die Dermatoskopie wahrscheinlich ist.

Ärgerlich ist es natürlich, wenn im Bedarfsfall kein dermatoskopisches Bild zur Verfügung steht. Es ist primär Aufgabe des Klinikers, dafür zu sorgen, dass das der Fall ist. Mit Einschränkungen ist es zwar auch möglich, nach der Exzision, also ex vivo, dermatoskopische Aufnahmen anzufertigen, eine Ex-vivoDermatoskopie kann jedoch eine intravital angefertigte Dermatoskopie nicht vollständig ersetzen, da einige wichtige Kriterien, wie z. B. Gefäßstrukturen, nicht mehr beobachtet werden können. Trotzdem wird die Ex-vivo-Dermatoskopie von manchen Dermatopathologen eingesetzt, nicht nur für eine dermatoskopisch-pathologische Korrelation, sondern auch, um beim Zuschneiden des Präparates die beste Schnittführung zu wählen [21].

\section{》) Sinnvoll ist eine Integration des dermatoskopischen Befun- des bei dermatopathologischen Zweifeln}

Wahrscheinlich ist es bei allen Läsionen, die aus diagnostischen Gründen entfernt werden, ratsam, ein dermatoskopisches und ein klinisches Bild anzufertigen, unabdingbar ist es aber bei Teilbiopsien. Der Arbeitsaufwand zur Anfertigung eines dermatoskopischen Bildes, das im $\mathrm{Zu}$ ge der Vorbereitung des Eingriffes auch von nichtärztlichem Personal aufgenommen werden kann, ist klein im Verhältnis zum Informationsgewinn.

\section{Praktische Hindernisse}

Es stellt sich also die Frage, warum die dermatoskopisch-pathologische Integration nicht überall standardmäßig bei melanozytären Läsionen durchgeführt wird. Ein wichtiges Hindernis ist die mangel- 
hafte Integration der Begutachtung externer Bilder in die Arbeitsabläufe des Dermatopathologen. Das dermatoskopische Bild muss während der Befundung schnell und zuverlässig zur Verfügung stehen, sodass keine Zeit für Bildsuche und Nachfragen verloren geht. Derzeit ist das in den seltensten Fällen gewährleistet. Man benötigt dazu eine eigene Infrastruktur, die von den meisten dermatopathologischen Labors nicht zur Verfügung gestellt wird.

Ein weiteres praktisches Hindernis ist die fehlende Bereitschaft von Dermatopathologen, sich mit der Dermatoskopie näher zu beschäftigen. Manche Dermatopathologen sind auch der Meinung, dass sie die Dermatoskopie im typischen Routinefall nicht benötigen und sich der Aufwand des Erlernens nicht lohnt. Der gleiche Einwand könnte aber auch für andere zusätzliche Untersuchungen wie die Immunhistochemie, FISH oder CGH vorgebracht werden, die auch im Routinefall nicht benötigt werden. Das mindert nicht den zusätzlichen Wert dieser Untersuchungen im Spezialfall. Außerdem ist die Dermatoskopie einfach zu erlernen und erweitert das Verständnis für melanozytäre Läsionen über die Routinebefundung hinaus.

Gelegentlich wird auch als Argument gegen eine Integration klinischer oder dermatoskopischer Befunde vorgebracht, dass sich der dermatopathologische Befund auf die Dermatopathologie beschränken soll. Nicht der Dermatopathologe, sondern der Kliniker soll alle Befunde $\mathrm{zu}$ einer abschließenden Diagnose integrieren. Dagegen wäre an sich gar nichts einzuwenden, hätte nicht der dermatopathologische Befund bei neoplastischen Veränderungen traditionellerweise einen Anspruch auf Letztgültigkeit. Bei entzündlichen Hauterkrankungen mag das anders sein, und oft ist es in diesen Fällen tatsächlich der Kliniker, der unter Einbeziehung aller möglichen Befunde eine abschließende Diagnose stellt [22]. Bei neoplastischen Veränderungen jedoch gilt das Wort des Pathologen und wird in der Regel vom Kliniker nicht mehr umgestoßen. Besteht ein Zweifel, wird der Pathologe gebeten, den Schnitt nochmals zu begutachten oder weitere Stufenschnitte anzufertigen, um die Zweifel auszuräumen. Bleibt der Pathologe bei seiner Diagnose, kann nur ein anderer Pathologe oder eine Gruppe von Pathologen den Befund revidieren, aber der Kliniker wird sich in der Regel davor hüten. Wenn die Dermatopathologie also ihren Anspruch auf Letztgültigkeit behalten will, muss sie auch andere, potenziell nützliche Informationen in den Befund integrieren, und dazu zählt ganz sicher die Dermatoskopie. Wer als Experte im Bereich der pathologischen Befundung melanozytärer Läsionen gelten möchte, muss den Blick auf das Ganze richten und kann die Dermatoskopie nicht einfach ignorieren, genauso wenig wie andere potenziell informative Zusatzinformationen.

\section{Fazit für die Praxis}

\section{- Die Dermatopathologie ist nach wie vor die wichtigste Untersuchung zur Dignitätsabklärung melanozytärer Läsionen. \\ - Aber nicht alle melanozytären Lä- sionen können anhand einer feinge- weblichen dermatopathologischen Untersuchung zweifelsfrei als gutar- tig oder bösartig klassifiziert werden. \\ - In unklaren Fällen kann die Einbe- ziehung des dermatoskopischen Befundes die Diagnosestellung er- leichtern. \\ - Eine dermatoskopisch-pathologische Korrelation ist allerdings nicht bei allen unklaren Läsionen hilfreich, sondern bevorzugt bei flachen pig- mentierten Läsionen.}

\section{Korrespondenzadresse}

\section{Prof. Dr. H. Kittler, MD}

Universitätsklinik für Dermatologie, Medizinische Universität Wien

Währinger Gürtel 18-20, 1090 Wien, Österreich harald.kittler@meduniwien.ac.at

Funding. Open access funding provided by Medical University of Vienna.

\section{Einhaltung ethischer Richtlinien}

Interessenkonflikt. H. Kittler gibt an, dass kein Interessenkonflikt besteht.

Dieser Beitrag beinhaltet keine vom Autor durchgeführten Studien an Menschen oder Tieren. Alle Patienten, die über Bildmaterial oder anderweitige Angaben innerhalb des Manuskripts zu identifizieren sind, haben hierzu ihre schriftliche Einwilligung gegeben. Im Falle von nicht mündigen Patienten liegt die Einwilligung eines Erziehungsberechtigten oder des gesetzlich bestellten Betreuers vor.

Open Access Dieser Artikel wird unter der Creative Commons Namensnennung 4.0 International Lizenz (http://creativecommons.org/licenses/by/4.0/deed. de) veröffentlicht, welche die Nutzung, Vervielfältigung, Bearbeitung, Verbreitung und Wiedergabe in jeglichem Medium und Format erlaubt, sofern Sie den/die ursprünglichen Autor(en) und die Quelle ordnungsgemäßnennen, einen Link zur Creative Commons Lizenz beifügen und angeben, ob Änderungen vorgenommen wurden.

\section{Literatur}

1. Sinz C, Tschandl P, Rosendahl C, Akay BN, Argenziano G, Blum A, Braun RP, Cabo H, Gourhant JY, Kreusch J, Lallas A, Lapins J, Marghoob AA, Menzies SW, Paoli J, Rabinovitz HS, Rinner C, Scope A, Soyer HP, Thomas L, Zalaudek I, Kittler H (2017) Accuracy of dermatoscopy for the diagnosis of nonpigmented cancers of the skin. J Am Acad Dermatol 77(6):1100-1109

2. Kittler $H$, Pehamberger $H$, WolffK, Binder M (2002) Diagnostic accuracy of dermoscopy. Lancet Oncol 3(3):159-165

3. Stevenson AD, Mickan S, Mallett S, Ayya M (2013) Systematic review of diagnostic accuracy of reflectance confocal microscopy for melanoma diagnosis in patients with clinically equivocal skin lesions. Dermatol Pract Concept 3(4):19-27

4. Xiong YQ, Mo Y, Wen YQ, Cheng MJ, Huo ST, Chen XJ, Chen Q (2018) Optical coherence tomography for the diagnosis of malignant skin tumors: a metaanalysis. JBiomed Opt 23(2):1-10

5. Tschandl P, Berghoff AS, Preusser M, BurgstallerMuehlbacher S, Pehamberger $\mathrm{H}$, Okamoto I, Kittler $\mathrm{H}$ (2013) NRAS and BRAF mutations in melanomaassociated nevi and uninvolved nevi. PLoS ONE 8;8(7):e69639

6. Elmore JG, Barnhill RL, Elder DE, Longton GM, Pepe MS, Reisch LM, Carney PA, Titus LJ, Nelson HD, Onega T, Tosteson ANA, Weinstock MA, Knezevich SR, Piepkorn MW (2017) Pathologists' diagnosis of invasive melanoma and melanocytic proliferations: observer accuracy and reproducibility study. BMJ 357:j2813

7. Lott JP, Elmore JG, Zhao GA, Knezevich SR, Frederick PD, Reisch LM, Chu EY, Cook MG, Duncan LM, Elenitsas R, Gerami P, Landman G, Lowe L, Messina JL, Mihm MC, van den Oord JJ, Rabkin MS, Schmidt B, Shea CR, Yun SJ, Xu GX, Piepkorn MW, Elder DE, Barnhill RL, International Melanoma Pathology Study Group (2016) Evaluation of the Melanocytic Pathology Assessment Tool and Hierarchy for Diagnosis (MPATH-Dx) classification scheme for diagnosis of cutaneous melanocytic neoplasms: results from 
the International Melanoma Pathology Study Group. J Am Acad Dermatol 75(2):356-363

8. Leary DE (2009) Between Peirce (1878) and James (1898): G. Stanley Hall, the origins of pragmatism, and the history of psychology. J Hist Behav Sci 45(1):5-20

9. Bauer J (2017) The molecular revolution in cutaneous biology: era of cytogenetics and copy numberanalysis. Jinvest Dermatol 137(5):e57-e59

10. Song J, Mooi WJ, Petronic-Rosic V, Shea CR, Stricker T, Krausz T (2011) Nevus versus melanoma: to FISH, or not to FISH. Adv Anat Pathol 18(3):229-234

11. Ferrara G, Argenyi Z, Argenziano G, Cerio R, Cerroni L, Di Blasi A, Feudale EA, Giorgio CM, Massone C, Nappi O, Tomasini C, Urso C, Zalaudek I, Kittler H, Soyer HP (2009) The influence of clinical information in the histopathologic diagnosis of melanocytic skin neoplasms. PLoSONE4(4):e5375

12. Ferrara G, Argenziano G, Giorgio CM, Zalaudek I, Kittler H (2009) Dermoscopic-pathologic correlation: apropos of six equivocal cases. Semin Cutan Med Surg 28(3):157-164

13. Massi D, De Giorgi V, Soyer HP (2001) Histopathologic correlates of dermoscopic criteria. Dermatol Clin 19(2):259-268

14. Argenziano G, Fabbrocini G, Carli P, De Giorgi V, Delfino M (1999) Clinical and dermatoscopic criteria for the preoperative evaluation of cutaneous melanoma thickness. J Am Acad Dermatol 40(1):61-68

15. Rosendahl C, Cameron A, McColl I, Wilkinson D (2012) Dermatoscopy in routine practice - 'chaos and clues.' Aust Fam Physician 41(7):482-487

16. Kittler $\mathrm{H}$, Pehamberger $\mathrm{H}$, Wolff $\mathrm{K}$, Binder $\mathrm{M}$ (2000) Follow-up of melanocytic skin lesions with digital epiluminescence microscopy: patterns of modifications observed in early melanoma, atypical nevi, and common nevi. J Am Acad Dermatol 43(3):467-476

17. Kittler H, Guitera P, Riedl E, Avramidis M, Teban L, Fiebiger M, Weger RA, Dawid M, Menzies S (2006) Identification of clinically featureless incipient melanoma using sequential dermoscopy imaging. Arch Dermatol 142(9):1113-1119

18. Tschandl P, Hofmann L, Fink C, Kittler H, Haenssle HA (2017) Melanomas vs. nevi in high-risk patients under long-term monitoring with digital dermatoscopy: do melanomas and nevi already differ at baseline? J Eur Acad Dermatol Venereol 31(6):972-977

19. Ackerman $A B$ (1994) Criteria for histopathologic diagnosis of melanoma, including melanoma in situ, in historical perspective. J Dermatol 21(11):872-874

20. Ritter A, Tronnier M, Vaske B, Mitteldorf C (2018) Reevaluation of established and new criteria in differential diagnosis of Spitz nevus and melanoma. Arch Dermatol Res. https://doi.org/10. 1007/s00403-018-1818-8

21. Amin K, Fraga GR (2012) Ex vivo dermoscopy of cutaneous biopsies for melanocytic neoplasms: a retrospective review of 517 cases with histopathologic correlation. Am J Dermatopathol 34(7):710-715

22. Cerroni L, Argenyi Z, Cerio R, Facchetti F, Kittler $\mathrm{H}$, Kutzner $\mathrm{H}$, Requena L, Sanqueza OP, Smoller B, Wechsler J, Kerl H (2010) Influence of evaluation of clinical pictures on the histopathologic diagnosis of inflammatory skin disorders. J Am Acad Dermatol 63(4):647-652

23. Kittler T (2015) Dermatoskopie, 2. Aufl. Facultas, Wien
Hier steht eine Anzeige. Springer 